\title{
Association between ovarian cancer and advanced endometriosis
}

\author{
MICHAIL MATALLIOTAKIS ${ }^{1}$, CHAROULA MATALLIOTAKI ${ }^{1}$, GEORGE N. GOULIELMOS ${ }^{2}$, \\ EVRIDIKI PATELAROU ${ }^{3}$, MARIA TZARDI $^{4}$, DEMETRIOS A. SPANDIDOS ${ }^{5}$, \\ AYDIN ARICI $^{6}$ and IOANNIS MATALLIOTAKIS ${ }^{1}$
}

\begin{abstract}
${ }^{1}$ Department of Obstetrics and Gynecology, Venizeleio General Hospital, 71409 Heraklion; ${ }^{2}$ Section of Molecular Pathology and Human Genetics, Department of Internal Medicine, School of Medicine, University of Crete, 71003 Heraklion;

${ }^{3}$ School of Health Sciences and Welfare, Technological Educational Institute of Crete, 71004 Heraklion; ${ }^{4}$ Department of Histopathology, University Hospital of Heraklion, Faculty of Medicine, 71110 Heraklion; ${ }^{5}$ Laboratory of Clinical Virology, Medical School, University of Crete, 71003 Heraklion, Crete, Greece; ${ }^{6}$ Department of Obstetrics, Gynecology and Reproductive Sciences, Yale University School of Medicine, New Haven, CT 06520, USA
\end{abstract}

Received February 1, 2018; Accepted March 15, 2018

DOI: $10.3892 / \mathrm{ol} .2018 .8287$

\begin{abstract}
We retrospectively analyzed clinicopathological data in two different countries over the past years on the association between ovarian endometriosis and ovarian carcinoma. Medical and pathological reports were evaluated from 1,000 patients with endometriosis from two different geographical areas. The prevalence and women characteristics of cases were analyzed. Endometriosis-associated ovarian cancer was present in $20(2 \%)$ cases, among the study subjects. The observed prevalence was $12(60 \%)$ for endometrioid carcinoma, 4 (20\%) for clear cell ovarian carcinoma, 2 (10\%) for serous and 2 (10\%) for mucinous adenocarcinoma. A higher proportion of endometrioid carcinoma cases were noted in comparison with other types $(\mathrm{P}<0.001)$. We found only $3 / 20(15 \%)$ postmenopausal cases. In all cases, we reported advanced stage of endometriosis (stage III or IV). Left-sided endometrioid carcinoma were notably more common than right-sided ones $(\mathrm{P}<0.001)$. In the majority of cases, malignant transformation of endometriosis was observed in endometrioid carcinoma or clear cell carcinoma of the ovary. Further research is required to establish the relationship between endometriosis and ovarian cancer.
\end{abstract}

\section{Introduction}

Endometriosis is an ordinary gynecological disorder that is characterized as the implantation of endometrium-like grandular and stromal cells beyond their normal location in

Correspondence to: Dr Michail Matalliotakis, Department of Obstetrics and Gynecology, Venizeleio General Hospital, Knossou Avenue 44, 71409 Heraklion, Crete, Greece

E-mail: mihalismat@hotmail.com

Key words: endometriosis-associated ovarian cancer, endometrioid carcinoma, clear cell ovarian carcinoma, mucinous adenocarcinoma, serous adenocarcinoma the uterus in 5 to $15 \%$ of all women. Although endometriosis is proven to be a benign disorder, clinical, histopathologic, epidemiologic and molecular data suggest that it does share common characteristics with the neoplastic process, including inflammatory processes and tissue invasion (1).

The association of endometriosis and ovarian cancer was initially reported by Sampson in 1925 (2). Ovarian cancer is considered to be the second most common gynecologic malignancy in developed countries (3). It has been found that endometriosis and invasive cancer are characterized by several common molecular and pathogenetic pathways, including inflammatory processes, induction of angiogenesis, proliferation and activation of invasion and metastasis, increased local estrogen production, similar cytokines and mediators, immune deregulation and resistance to apoptosis $(4,5)$. Endometriosis-associated ovarian cancer has been evidenced to deviate from non-endometriosis-associated ovarian cancer in many of its major biological characteristics (6). Of interest, endometriosis-associated ovarian cancer was found to exhibit better survival rate and lower-grade tumors than ovarian cancer not associated with endometriosis (7).

Transitional zones of endometriosis, ranging from benign to atypical and adjacent to ovarian malignancy have been well documented. Several research studies have specifically addressed the risk of certain types of ovarian cancers in patients with endometriosis. According to the literature, the risk of ovarian cancer among women with endometriosis has increased by 30 to $40 \%$, compared to women that do not suffer from this condition. Clear cell endometrial carcinoma is one of the most common types of ovarian cancer linked to endometriosis (8-10). Moreover, the risk of ovarian cancer increases for women who experience fewer pregnancies or suffer from infertility (11). Nonetheless, novel research from large case-controls and prospective cohort studies has managed to demonstrate the potential of endometriosis to become malignant transformation.

The observation of 20 cases of endometriosis in our 1,000 patients has led us to update the literature and to make various clinical observations. 
Table I. Clinicopathological characteristics and frequency of ovarian cancer cases with endometriosis.

\begin{tabular}{|c|c|c|c|c|}
\hline Variables & Age (years) & Stage of carcinoma & Stage of endometriosis & Site of origin of ovarian cancer \\
\hline \multicolumn{5}{|c|}{$\begin{array}{l}\text { Endometrioid carcinoma } \\
{[12 / 20(60 \%)]}\end{array}$} \\
\hline 1 & 42 & IC & IV & Left \\
\hline 2 & 39 & IA & III & Right \\
\hline 3 & 40 & IA & III & Left \\
\hline 4 & 48 & IA & IV & Left \\
\hline 5 & 61 & IIA & IV & Left \\
\hline 6 & 55 & IC & III & Right \\
\hline 7 & 45 & IA & III & Left \\
\hline 8 & 44 & IIA & III & Left \\
\hline 9 & 47 & IIA & III & Left \\
\hline 10 & 35 & IIA & III & Left \\
\hline 11 & 38 & IIA & IV & Left \\
\hline 12 & 49 & IIC & IV & Right \\
\hline \multicolumn{5}{|c|}{$\begin{array}{l}\text { Clear cell ovarian carcinoma } \\
{[4 / 20(20 \%)]}\end{array}$} \\
\hline 1 & 42 & IA & IV & Left \\
\hline 2 & 52 & IC & III & Right \\
\hline 3 & 39 & IC & IV & Left \\
\hline 4 & 51 & IIA & III & Left \\
\hline \multicolumn{5}{|c|}{$\begin{array}{l}\text { Serous adenocarcinoma } \\
{[2 / 20(10 \%)]}\end{array}$} \\
\hline 1 & 34 & IA & III & Right \\
\hline 2 & 56 & IIC & III & Left \\
\hline \multicolumn{5}{|c|}{$\begin{array}{l}\text { Mucinous adenocarcinoma } \\
{[2 / 20(10 \%)]}\end{array}$} \\
\hline 1 & 44 & IA & III & Left \\
\hline 2 & 48 & IIA & III & Left-right \\
\hline
\end{tabular}

\section{Patients and methods}

Clinical, surgical and pathological records were obtained from 550 consecutive women with endometriosis undergoing surgical treatment between January-June 1996 and July-December 2005, from the Yale University Hospital and 450 cases between 1990 and 2015, from the Department of Obstetrics and Gynecology of the University of Crete and the Department of Venizeleio General Hospital of Crete. The Human Committee of Yale University School of Medicine approved the study for this evaluation (HIC no. 12590) and the Ethics Committee for Human Research of Venizeleio Hospital has approved the respective protocol (ECHR no. 46/6686). Informed consent was provided by the participants.

The stage of endometriosis was scored according to the revised classification of the American Fertility Society (12) and the biopsy specimen was taken to confirm the diagnosis histologically. The criteria of FIGO were used in order to classify the clinicopathologic features of the ovarian carcinoma (13). Data were recorded, including age, parity, symptoms, stage of endometriosis, side and stage of ovarian tumors. Information on the histological type of ovarian cancer was retrieved from pathological records. The frequency of left- and right-sided ovarian cancer was analyzed with the $\chi^{2}$ test to compare observed and expected events.

\section{Results}

Clinicopathological characteristics. A total of 20 ovarian cancer cases were noted among the study subjects (Table I). A significant predominance of endometrioid carcinoma cases was documented in comparison with other types $(\mathrm{P}<0.001)$. Table I shows the frequency of different types of ovarian malignancies. Cases of endometrioid cancer were the most common [12 (60\%)], followed by clear cell ovarian cancer [4 (20\%)], serous adenocarcinoma [2 (10\%)] and mucinus adenocarcinoma [2(10\%)]. The mean age of all affected women was $45.5 \pm 6.5$ years.

The stage of all ovarian carcinomas was extended until IIC, and all of the cases were in an advanced stage of endometriosis (stage III or IV) (Table I). Finally, the women with endometrioid cancer were observed with a left-sided spreading of the malignancy in $75 \%$ of cases $(9 / 12)$, notably higher compared with the right-sided ones $[25 \%(3 / 12)](\mathrm{P}<0.001)$. 


\section{Discussion}

In the ovaries, endometrioid cancer represents the most known example of an epithelial malignancy arising from endometriosis, followed by clear cell carcinoma. On the other hand, serous and mucinous adenocarcinomas are prevalent in ovarian cancers unrelated to endometriosis. Endometrioid cancer (up to $60 \%$ of cases) and clear cell carcinoma (up to $15 \%$ of cases) predominate in higher proportions than have been observed among ovarian cancers in general (10-20\% and $3-10 \%$, respectively), indicating the specific connection between these cancer types and endometriosis. Despite the fact that adjacent endometriosis may reveal metaplastic and atypical alterations, a certain transition from endometriosis to endometrioid cancer can be estimated in $5-10 \%$ of women $(1,9,10,14)$. According to large cohort and case control studies, the prevalence of ovarian cancer that appears in patients with endometriosis is greater than sporadic ovarian cancer in the general population $(9,15-17)$. This statement is of great interest as epithelial ovarian malignancy accounts for $80-90 \%$ of all ovarian cancers and is the major cause of death from gynecologic malignancies. The overall 5-year survival rate remains low, despite significant improvements in the management of these cases.

To elucidate the molecular mechanism underlying the malignant transformation of endometriosis, and considering the important role of genetics in the development of both endometriosis and ovarian cancer, many studies thus far have attempted to evaluate whether endometriosis-associated genetic alterations or gene polymorphisms increase the risk of ovarian cancer. Notably, strong evidences of a genetic link between endometriosis and ovarian cancer have been detected thus far. Loss of heterozygosity at 10q23.3 and mutations leading to functional inactivation of the PTEN gene as well as ARID1A, PIK3CA, CTNNB1 ( $\beta$-catenin), TP53 and $K$-ras gene mutations have been shown to contribute to endometriosis-associated ovarian cancer (18-21). Furthermore, rs11651755 SNP of HNF1B gene, rs3754496 located near WNT4 gene and rs1333052 located adjacent to $C D K N 2 B$ gene has been suggested to be causally involved in the pathogenetic pathway leading from endometriosis to ovarian cancer $(22,23)$. Recently, application of the next-generation sequencing has led to the identification of more shared genetic susceptibility loci between endometriosis and all histotypes of ovarian cancer, a finding that is promising regarding the delineation of the underlying molecular pathways in the future (24).

We previously reported (25) a case with endometriosisassociated stage AI clear cell ovarian carcinoma in a woman with IVF-ET treatments in the Yale series. This unusual case serves as a reminder that clear cell adenocarcinoma may coexist with endometriosis. Moreover, we investigated the familial risk of ovarian cancer in women with endometriosis diagnosed by laparoscopy, based on the medical records of the Yale New Haven Hospital between 1996 and 2002. In that study, we found that the proportion of patients with positive family history of ovarian cancer in first and second degree relatives was notably increased in women with endometriosis compared with the control group (26). In the current study, we detected $20(2 \%)$ cases of endometriosis-associated ovarian cancer in
1,000 women with endometriosis. We reported endometrioid cancer $(60 \%)$ more frequently, followed by clear cell carcinoma (20\%), serous and mucinous (20\%) adenocarcinomas. These results suggest that women with endometriosis have an increased risk for certain types of ovarian cancers. The prevalence of ovarian malignancy in patients with endometriosis seems to be elevated compared to the general population. One reason for this coexistence of both conditions may be that both diseases share risk factors such as infertility and nulliparity. Alternatively, related hormonal imbalances may be responsible for the development of both conditions (27).

Notably, several observations, mainly epidemiologic, suggest that females who have been exposed to chronic pelvic inflammation seem to be at an elevated risk for the development of epithelial ovarian cancer (28). In our series, we found unilateral endometrioid cancer more frequently in the left (75\%) than in the right ovary (25\%). Our results are in agreement with Vercellini et al who investigated the distribution of early stage epithelial ovarian cancers. This group documented that the proportion of the left-sided lesions was prominent in the endometrioid type compared to other types (1). This observation suggests that the pathogenesis of endometrioid cancer is marked from that of other epithelial malignancies of the ovary. Moreover, it is hypothesized that a correlation may exist between ovarian endometriomas and malignant endometrioid tumors. Finally, we observed only three postmenopausal women with ovarian cancer. This finding suggests that younger ages with endometriosis and ovarian cancer are affected.

In conclusion, our findings indicate that women with endometriosis have an increased risk for several types of ovarian cancers, more frequently endometrioid carcinoma. Further research is required to establish the relationship between endometriosis and malignancies.

\section{Acknowledgements}

We would like to thank all the clinicians and pathologists for providing the data used in this study.

\section{Funding}

No funding was received.

\section{Availability of data and materials}

The datasets used and/or analyzed during the current study are available from the corresponding author on reasonable request.

\section{Authors' contributions}

Michail and Ioannis Matalliotakis conceived and designed the study. Aydin Arici, Tzardi Maria and Ioannis Matalliotakis obtained the data. Michail Matalliotakis, George N. Goulielmos, Evridiki Patelarou, Charoula Matalliotaki, and Demetrios A. Spandidos analysed and interpreted the data. Michail and Charoula Matalliotakis drafted the manuscript. Michail Matalliotakis, George N. Goulielmos, Charoula Matalliotaki, Evridiki Patelarou, and Demetrios A. Spandidos critically revised the manuscript. 


\section{Ethics approval and consent to participate}

The Human Committee of Yale University School of Medicine approved the study for this evaluation (HIC no. 12590) and the Ethics Committee for Human Research of Venizeleio Hospital has approved the respective protocol (ECHR no. 46/6686). Informed consent was obtained from all the participants.

\section{Consent for publication}

Not applicable.

\section{Competing interests}

Demetrios A. Spandidos is the Editor-in-Chief for the journal, but had no personal involvement in the reviewing process, or any influence in terms of adjudicating on the final decision, for this article.

\section{References}

1. Vercellini P, Scarfone G, Bolis G, Stellato G, Carinelli S and Crosignani PG: Site of origin of epithelial ovarian cancer: The endometriosis connection. BJOG 107: 1155-1157, 2000.

2. Sampson JA: Endometrial carcinoma of the ovary, arising in endometrial tissue in that organ. Arch Surg 10: 1-72, 1925.

3. Vaughan S, Coward JI, Bast RC Jr, Berchuck A, Berek JS, Brenton JD, Coukos G, Crum CC, Drapkin R, Etemadmoghadam D, et al: Rethinking ovarian cancer: Recommendations for improving outcomes. Nat Rev Cancer 11: 719-725, 2011.

4. Guo SW: Endometriosis and ovarian cancer: Potential benefits and harms of screening and risk-reducing surgery. Fertil Steril 104: 813-830, 2015

5. Hanahan D and Weinberg RA: Hallmarks of cancer: The next generation. Cell 144: 646-674, 2011

6. Erzen M, Rakar S, Klancnik B and Syrjänen K: Endometriosisassociated ovarian carcinoma (EAOC): An entity distinct from other ovarian carcinomas as suggested by a nested case-control study. Gynecol Oncol 83: 100-108, 2001.

7. Kumar S, Munkarah A, Arabi H, Bandyopadhyay S, Semaan A, Hayek K, Garg G, Morris R and Ali-Fehmi R: Prognostic analysis of ovarian cancer associated with endometriosis. Am J Obstet Gynecol 204: 63.e1-63.e7, 2011.

8. Birrer MJ: The origin of ovarian cancer - Is it getting clearer? N Engl J Med 363: 1574-1575, 2010.

9. Kappou D, Matalliotakis M, Sifakis S, Ziogos E and Matalliotakis I: The association of endometriosis to various types of malignancies: A comprehensive review of current knowledge and future directions. In: New Developments in Endometriosis. Matalliotakis I and Arici A (eds). CreateSpace, pp416-456, 2011.

10. Wilbur MA, Shih IM, Segars JH and Fader AN: Cancer implications for patients with endometriosis. Semin Reprod Med 35: 110-116, 2017.

11. Greene AD, Lang SA, Kendziorski JA, Sroga-Rios JM, Herzog TJ and Burns KA: Endometriosis: Where are we and where are we going? Reproduction 152: R63-R78, 2016.
12. American Fertility Society: Revised American Fertility Society classification of endometriosis: 1985. Fertil Steril 43: 351-352, 1985.

13. Creasman WT: Announcement, FIGO stages: 1988 revisions. Gynecol Oncol 35: 125-127, 1989.

14. Prefumo F, Todeschini F, Fulcheri E and Venturini PL: Epithelial abnormalities in cystic ovarian endometriosis. Gynecol Oncol 84: 280-284, 2002.

15. Brinton LA, Lamb EJ, Moghissi KS, Scoccia B, Althuis MD, Mabie JE and Westhoff CL: Ovarian cancer risk associated with varying causes of infertility. Fertil Steril 82: 405-414, 2004

16. Brinton LA, Westhoff CL, Scoccia B, Lamb EJ, Althuis MD, Mabie JE and Moghissi KSP: Causes of infertility as predictors of subsequent cancer risk. Epidemiology 16: 500-507, 2005.

17. Melin A, Sparén P, Persson I and Bergqvist A: Endometriosis and the risk of cancer with special emphasis on ovarian cancer. Hum Reprod 21: 1237-1242, 2006.

18. Sato N, Tsunoda H, Nishida M, Morishita Y, Takimoto Y, Kubo T and Noguchi M: Loss of heterozygosity on 10q23.3 and mutation of the tumor suppressor gene PTEN in benign endometrial cyst of the ovary: Possible sequence progression from benign endometrial cyst to endometrioid carcinoma and clear cell carcinoma of the ovary. Cancer Res 60: 7052-7056, 2000.

19. Singer G, Kurman RJ, Chang H-W, Cho SK and Shih IeM: Diverse tumorigenic pathways in ovarian serous carcinoma. Am J Pathol 160: 1223-1228, 2002.

20. Levanon K, Crum C and Drapkin R: New insights into the pathogenesis of serous ovarian cancer and its clinical impact. J Clin Oncol 26: 5284-5293, 2008.

21. Wiegand KC, Shah SP, Al-Agha OM, Zhao Y, Tse K, Zeng T, Senz J, McConechy MK, Anglesio MS, Kalloger SE, et al: ARID1A mutations in endometriosis-associated ovarian carcinomas. N Engl J Med 363: 1532-1543, 2010.

22. Lee AW, Templeman C, Stram DA, Beesley J, Tyrer J, Berchuck A, Pharoah PP, Chenevix-Trench G, Pearce CL and Ness RB; Ovarian Cancer Association Consortium: Evidence of a genetic link between endometriosis and ovarian cancer. Fertil Steril 105: 35-43.el, 10, 2016.

23. Burghaus S, Fasching PA, Häberle L, Rübner M, Büchner K, Blum S, Engel A, Ekici AB, Hartmann A, Hein A, et al: Genetic risk factors for ovarian cancer and their role for endometriosis risk. Gynecol Oncol 145: 142-147, 2017.

24. Lu Y, Cuellar-Partida G, Painter JN, Nyholt DR, Morris AP, Fasching PA, Hein A, Burghaus S, Beckmann MW, Lambrechts D, et al; Australian Ovarian Cancer Study; International Endogene Consortium (IEC): Shared genetics underlying epidemiological association between endometriosis and ovarian cancer. Hum Mol Genet 24: 5955-5964, 2015.

25. Matalliotakis I, Mahutte NG, Koukoura O and Arici A: Endometriosis associated with stage IA clear cell ovarian carcinoma in a woman with IVF-ET treatments in the Yale Series. Arch Gynecol Obstet 274: 184-186, 2006.

26. Matalliotakis IM, Cakmak H, Krasonikolakis GD, Dermitzaki D, Fragouli Y, Vlastos G and Arici A: Endometriosis related to family history of malignancies in the Yale series. Surg Oncol 19: 33-37, 2010.

27. Jimbo H, Yoshikawa H, Onda T, Yasugi T, Sakamoto A and Taketani Y: Prevalence of ovarian endometriosis in epithelial ovarian cancer. Int J Gynaecol Obstet 59: 245-250, 1997.

28. Ness RB: Endometriosis and ovarian cancer: Thoughts on shared pathophysiology. Am J Obstet Gynecol 189: 280-294, 2003. 